\title{
Enhanced optical transmission through planar metallic films via excitation of surface plasmons
}

\author{
Richard Blaikie ${ }^{\dagger}$, Ling Lin and Roger Reeves \\ MacDiarmid Institute for Advanced Materials and Nanotechnology, \\ University of Canterbury, Christchurch 8020, New Zealand \\ ${ }^{\dagger}$ Email: r.blaikie@elec.canterbury.ac.nz
}

\begin{abstract}
A study on extraordinary optical transmission through planar metallic films closely coupled to periodic sub-wavelength structures is presented. The measured spectral positions of transmission maxima match well with the surface plasmon polariton (SPP) dispersion relations. The experimental results are further supported by rigorous coupled wave analysis (RCWA) simulations. The exceptional transmission is a result of coupling between the localized surface plasmon (LSP) mode existing in periodic sub-wavelength structures and the SPP modes on the planar metal films.
\end{abstract}

Keywords-surface plasmons;enhanced transmission;metallic thin films;periodic structures.

\section{INTRODUCTION}

Surface-plasmon-enhanced optical transmission through thin metallic films is currently a rapid and expanding field of research. It opens possibilities of developing optical elements at the nanoscale, without the constraints of diffraction limits encountered in conventional optics. To date works in this area have been devoted to metallic films with periodic subwavelength patterns on [1-4] or through the film [5-10]. However the fact that the SPs and the sub-wavelength features that generate them co-exist on the same film (or films) tends to confound the analysis somewhat. We report here on the study of the optical transmission properties of unpatterned planar metallic films closely coupled to periodic subwavelength structures, showing that planar film can also give rise to SP enhanced transmission, providing there is some means of generating the necessary surface plasmon (SP) modes.

\section{Structure Design}

The schematics of the structures we have studied experimentally are shown in Fig. 1(a). They consists of a glass substrate, a thin metallic film (Ag) and a dielectric spacer layer $\left(\mathrm{SiO}_{2}\right)$ with periodic subwavelength structures (gratings or hole arrays) on the top. The periodicity presented on top of the structure provides the required in-plane wavevector for optical excitation of surface plasmon polaritons (SPPs) on the interfaces of the planar metal film. This configuration offers great flexibility in controlling the optical properties of the structure through adjusting the dimensions of each individual components.

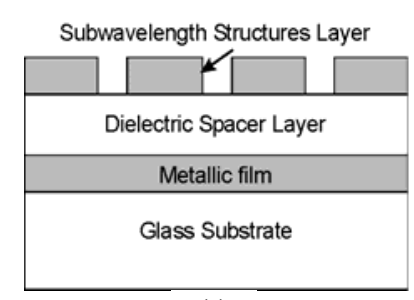

(a)

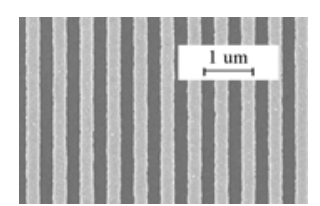

(b)

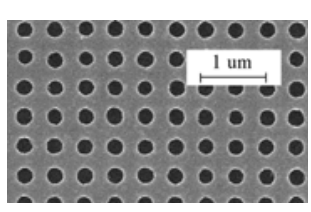

(c)
Figure 1. (a) A schematic diagram of the structure studied. (b) A SEM image of a fabricated transmission grating structure. (c) A SEM image of a fabricated hole array structure.

\section{EXPERIMENTAL METHODS}

\section{A. Sample Fabrication}

The samples were fabricated on $22 \mathrm{~mm} \times 22 \mathrm{~mm} \times 0.5 \mathrm{~mm}$ BK7 glasses. The fabrication process started from RF sputtering a layer of $35 \mathrm{~nm}$-thick $\mathrm{Ag}$ followed by a layer of $200 \mathrm{~nm}$-thick $\mathrm{SiO}_{2}$ on to the cleaned substrates. A four-layer imaging scheme is then deposited onto the $\mathrm{SiO}_{2}$ layer for subwavelength structure generation. This includes a $50 \mathrm{~nm}$ thick PMMA layer, a $150 \mathrm{~nm}$-thick anti-reflection coating (ARC), a $40 \mathrm{~nm}$-thick $\mathrm{SiO}_{2}$ layer and a $160 \mathrm{~nm}$-thick i-line photoresist on top. The arrays patterns were firstly defined onto the photoresist layer using interference lithography (IL) technique. Gratings patterns involved only a single exposure; hole arrays required double exposures and patterns were initially defined as dot arrays. The lattice constants and the dimensions of the elements of the patterns were controlled by varying the exposure conditions. Following exposure and development of the photoresist, the defined patterns were transferred through the four-layer stack via two separate reactive ion etching (RIE) process for $\mathrm{SiO}_{2}$ and ARC/PMMA. In this step, the thin layer of $\mathrm{SiO}_{2}$ between the $\mathrm{ARC}$ and photoresist serves as the hard etching mask for the following 
ARC/PMMA etching so that a slightly undercut profile can be produced. After dry etching, a layer of $35 \mathrm{~nm}$-thick Ag film was thermally evaporated onto the sample; the final step is lift-off, in which the patterns in the image stack were transferred on top of the $\mathrm{SiO}_{2}$ spacer with an inverted profile. The bottom PMMA layer in image stack is a sacrificial layer for easy stripping of the ARC during this final lift-off step. Fig. 1(b) and 1(c) show scanning electron microscope (SEM) images of fabricated grating and hole-array structures, respectively.

\section{B. Optical Characterization}

The zero-order transmission spectra of the samples under linear polarized light are measured using a custom-built spectrophotometer system. The system was built up based around a GSA/McPherson 1 metre focal length monochromator fitted with a prism predisperser. The output of the monochromator is collimated onto the detector using off-axis parabolic mirrors and the samples can be mounted at the required angle to the collimated monochromatic beam. The detectors are $10 \mathrm{~mm}^{2}$ silicon photodiodes for the transmittance measurements. The measurements were made over the wavelength range $550 \mathrm{~nm}$ to $850 \mathrm{~nm}$ at $1 \mathrm{~nm}$ intervals with a bandwidth of $0.5 \mathrm{~nm}$

\section{RESULTS AND DISCUSSION}

\section{A. Experimental Results}

Fig. 2(a) shows a measured transmission spectrum of a fabricated sample at normal incidence (solid line). The subwavelength structure on top of the sample is a hole array with period of $P_{h}=485 \mathrm{~nm}$. The diameter of the circular holes is $D \approx 220 \mathrm{~nm}$. For comparison, we also produced a sample consisting of a $35 \mathrm{~nm}$ planar $\mathrm{Ag}$ film, $200 \mathrm{~nm}$ of $\mathrm{SiO}_{2}$ and a $35 \mathrm{~nm}$ planar Ag film on the BK7 substrate. The measured transmittance of this reference sample is shown as the dashed line in Fig. 2(a). The simulation results for these samples obtained from a rigorous coupled-wave analysis (RCWA) [11] based commercial grating analysis software, G-Solver [12], are shown in Fig. 2(b). In the simulation, the circular holes were replaced with rectangular holes of same surface areas to reduce the computation time. The complex refractive indices of $\mathrm{Ag}$ used in the simulations were attained from linear interpolation of the tabulated data in Ref [13]; the refractive indices of the $\mathrm{SiO}_{2}$ and $\mathrm{BK} 7$ glass are set to 1.454 and 1.512 , respectively.

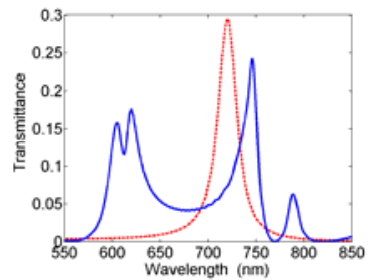

(a)

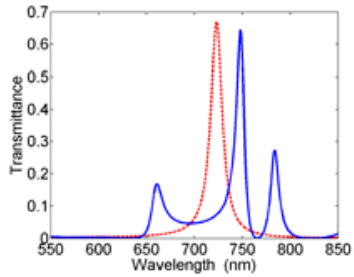

(b)
Figure 2. Transmission spectra of a planar $\mathrm{Ag}$ film- $\mathrm{SiO}_{2}$-planar $\mathrm{Ag}$ film structure (dashed line) and a planar $\mathrm{Ag}$ film- $\mathrm{SiO}_{2}-\mathrm{Ag}$ hole arrays structure (solid line): (a) measurements; (b) simulation results.
Three distinct peaks appear in the transmission spectra of the structures studied here, and the maximum transmission occurs for the centre peak (the second peak). The measured transmittance is around $25 \%$. Clearly this peak does not arise from the Fabry-Perot resonance as its spectral position, the shape and the linewidth are rather different from the peak produced by the planar Fabry-Perot cavity. The periodic subwavelength structure presented on top of the sample has dramatically modified the transmission properties of the cavity, even though the holes only occupy $\sim 20 \%$ of the surface area.

Fig. 3(a) shows the normal-incidence transmission spectra of two fabricated samples with rectangular transmission gratings on top. The grating periods on these two samples are $P_{1}=475 \mathrm{~nm}$ (solid line) and $P_{2}=485 \mathrm{~nm}$ (dashed line) and the slit widths in the gratings are $D_{1} \approx 215 \mathrm{~nm}$ and $D_{2} \approx 220 \mathrm{~nm}$. The electric field of the incident light is perpendicular to the gratings in these measurements (TM polarization). The corresponding simulation results are shown in Fig. 3(b). We observed that position of the broad peak appearing in the shorter wavelength side of the spectrum (the first peak) is not affected by the variation of the grating period. In contrast, the position of the center peak and the one that follows it (the third peak) are closely related to the period of the gratings. The period dependence in the spectral positions of the second and the third peak is confirmed by examining the spectra displayed in Fig. 2. In this figure, the lattice constant of the hole arrays on the measured sample is $485 \mathrm{~nm}$, which is the same as the period of the gratings on one of the sample used for Fig. 3. The measured centre wavelengths of the second peaks for these two samples are $746 \mathrm{~nm}$ and $748 \mathrm{~nm}$. The measured spectral separation between the second and the third peak for the sample with hole arrays is $44 \mathrm{~nm}$ and that for the sample with gratings is $47 \mathrm{~nm}$. These values are very close to each other, although the topologies of the subwavelength structure on top of two samples are completely different.

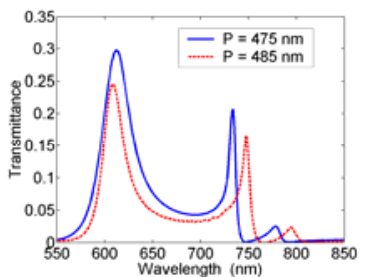

(a)

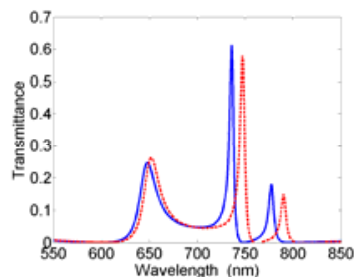

(b)
Figure 3. Transmission spectra of two fabricated samples with Ag gratings on top. The periods of the gratings are $475 \mathrm{~nm}$ and $485 \mathrm{~nm}$. (a) measurements; (b) simulation results.

It is well know that for a thin metal film $(<100 \mathrm{~nm})$ sandwiched between two dielectric media of same or similar dielectric constants, SPP waves on two interfaces of the metal film will interfere, resulting in a splitting of SPPs into a high frequency $\omega^{+}$mode and a low frequency $\omega^{-}$mode. We calculated the dispersion relations of these two modes on a $35 \mathrm{~nm}$-thick planar Ag film with BK7 glass bounded on both sides by solving the implicit dispersion relation (1) and (2) [14] below numerically with the aid of (3). 


$$
\begin{gathered}
\omega^{+}: \varepsilon_{m} k_{z d}+\varepsilon_{d} k_{z m} \tanh \left(k_{z m} l / 2 j\right)=0, \\
\omega^{-}: \varepsilon_{m} k_{z d}+\varepsilon_{d} k_{z m} \tanh \left(k_{z m} l / 2 j\right)=0 \\
k_{s p}^{2}+k_{z i}^{2}=\varepsilon_{i}(\omega / c)^{2}, \quad i=m, d,
\end{gathered}
$$

where: $\varepsilon_{m}$ and $\varepsilon_{d}$ are the dielectric constants of the metal and its surrounding dielectrics, respectively; $l$ is the thickness of the metal film; $k_{z m}$ and $k_{z d}$ are wavevectors in the metal and the dielectric with direction normal to the interface, respectively; $j^{2}=-1 ; k_{s p}$ is the wavevector of $\omega^{+}$or $\omega^{-}$along the interfaces; $\omega$ is the angular frequency of the incident light and $c$ is the speed of light in vacuum. It is also necessary to apply the dispersion relation for optical excitation of SPPs by a periodic structure,

$$
k_{s p}=(\omega / c) \sin \theta+2 m \pi / P
$$

where $\theta$ is the angle of incidence, $m$ is an integer for the mode index and $P$ is the lattice constant of the periodic structure. In the case of $m=1, \lambda_{\omega+}=747 \mathrm{~nm}$ and $\lambda_{\omega-}=797 \mathrm{~nm}$ at $P=$ $485 \mathrm{~nm}$ and $\lambda_{\omega^{+}}=733 \mathrm{~nm}$ and $\lambda_{\omega^{-}}=783 \mathrm{~nm}$ at $P=475 \mathrm{~nm}$. The wavelengths of the second and the third transmission peaks from both measurements and simulations closely match these numbers, which strongly suggests that the second and the third peak in the transmission spectra are attributable to the excitation of $\omega^{+}$and $\omega^{-}$mode on the planar $\mathrm{Ag}$ film, respectively. Because the electric fields associated with $\omega^{+}\left(\omega^{-}\right)$ modes inside the metal film decreases (increases) as the film thickness reduces [14], the rate of the internal damping of the $\omega^{+}$mode is smaller than that of the $\omega^{-}$mode for the thickness of the planar film studied here. Therefore we observed the higher transmission efficiency and narrower linewidth of the $\omega^{+}$mode (the second peak) than that of the $\omega^{-}$mode (the third peak) for a given structure.

\section{B. Simulations}

To further analyze the transmission properties of the structures, we studied their spectral response under different excitation condition. Fig. 4(a) shows the simulated transmission spectra of a sample with gratings period of $485 \mathrm{~nm}$ under TM- and TE-polarized light at 5 degrees off normal incidence. The corresponding simulation results for the sample with hole-array on top are displayed in Fig. 4(b). We observed that for both structures the shape of SPP related peaks have changed dramatically under TM-polarized light due to SPPs gaining extra momentum from the incident light at offnormal incidence; for the structure with gratings the SPP related peaks disappeared under TE-polarized light. These responses are consistent with the nature of the SPPs. Interestingly there is no noticeable modification in the transmission spectrum of the structure with the hole-array layer on top under TE-polarized light while varying the angle of incidence. While this seems to contradict the nature of SPPs at first sight, it can be explained by considering the excitation of the localized surface plasmons (LSPs) - the collective excitation of localization charge oscillations at the edges of the holes by the incident light [15]: the enhanced electric field extends to the planar Ag film underneath and induces the required surface charges to launch SPP waves on the planar film. Since the wavevector of the incident light and the oscillations of the E-field are out of phase for TE-polarized light, the wavelength of the SPP modes are unaffected by the incident light and solely depends on the lattice constant of the hole arrays.

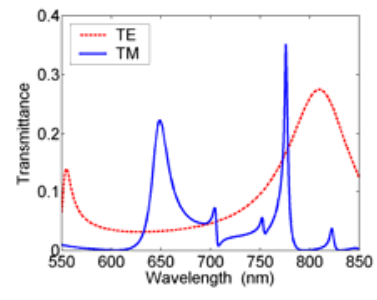

(a)

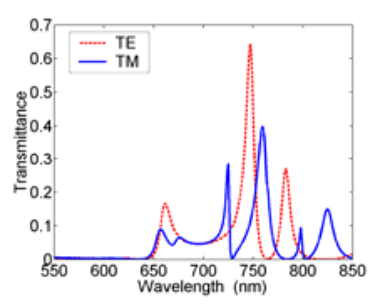

(b)
Figure 4. Simulated transmission spectra of the structures under TM- and TE-polarized light at 5 degrees of incidence. (a) structue with gratings on top; (b) structure with hole arrays on top.

There is a relatively broad peak on the shorter wavelength side of the transmission spectra (the first peak). We consider that this peak is caused by the excitation of a waveguide mode within the structure. Simulations (results not show here) reveal that the spectral position and amplitude of this peak respond sensitively to the thickness and the optical constants of the dielectric spacer, as well as the opening area of the subwavelength structure layer. Comparing Fig. 3(b) and Fig. 4(b), we notice that the features of this peak in the transmission spectrum is almost untouched for a small variation in the angle of incidence under TM-polarized light whereas this change causes noticeable modification on the appearances of the other two; changing polarization of the incident light from TM to TE at oblique incidence alters the position and amplitude of this peak but its overall shape remains. These responses reflect the waveguide mode nature of this peak.

\section{Effects of Subwavelength Structure Topology on the Enhanced Transmission}

Both grating and hole-array structures show the ability to produce SP-enhanced optical transmission and the overall characteristics of the transmission spectra of these two devices are very similar: three peaks arise from the excitation of a waveguide mode, a high frequency and a low frequency SPP mode and the spectral positions of the SPP-related peaks are governed by the lattice constant of the subwavelength structure presented on top of the device. However, there are also subtle differences between the spectral features of two arrangements and we are mainly interested in the aspects of the amplitude and the linewidth of SPP related peak. By analyzing the origins of these discrepancies, we obtained a clearer picture of the mechanisms behind the exceptional transmission properties of the structure studied here.

Generally the transmission efficiency of the high frequency SPP related peak is higher for the samples with hole-array layer on top and the linewidth of this mode is broader in such an arrangement. The simulation result for the structure with hole 
arrays under TE-polarized light (Fig. 4(b)) indicates that in addition to the excitation of SPPs on the planar Ag film, the excitation of LSPs in the subwavelength structure layer also plays an important role in the enhanced transmission. Because the LSPs are non-propagating modes and confined to the subwavelength structure, the strength of the excitation and its frequency are determined by the geometries of the subwavelength structure. The field intensity of LSPs is higher in hole arrays than that in gratings due to the former being a two-dimensional arrangement. This result in enhanced coupling between SPPs and LSPs in the structure with holearray layer on top, hence the higher transmission level. The drawback of using the two-dimensional arrangement on top of the structure is that it introduces additional damping to the system. There are two damping processes that exist in the structure: the internal damping and radiation damping. The former is due to the finite electrical conductivity of the metal at optical frequencies, its strength increases with the rising intensity of the electric field; the latter is due to scattering agents (surface roughness, subwavelength structures) in the system and its intensity is proportional to the scatterer number. Therefore, the total damping in the structure with the holearray layer is higher than that in the structure with a grating layer, resulting in the broader linewidth.

\section{CONCLUSION}

We have demonstrated that the enhanced optical transmission can be achieved through the planar metallic films by closely coupling the films to subwavelength structures. The results from the experiments and the theoretical calculations confirm that the exceptional transmission is due to excitation of surface plasmon polariton modes on two interfaces of the planar metal films. The RCWA based simulations reveal that overall transmission property of the structure is a result of the couplings between the incident light, SPP modes and LSP mode within the structure. The strength and the linewidth of the surface plasmons enhanced transmission peaks are determined by the interaction between LSP and SPPs.

\section{ACKNOWLEDGMENT}

The authors wish to thank Helen Devereux and Gary Turner for their technical support, Dr. Damian Carder for assistance with UV laser and John Hamlin for assistance with optical measurements.

\section{REFERENCES}

[1] S. A. Darmanyan and A. V. Zayats, "Light tunneling via resonant surface plasmon polariton states and the enhanced transmission of periodically nanostructured metal films: An analytical study," Physical Review B, vol. 67,pp. art.no. 035424, 2003.

[2] D. Gerard, L. Salomon, F. de Fornel, and A. V. Zayats, "Ridge-enhanced optical transmission through a continuous metal film," Physical Review B, vol. 69, pp. art. no.113405, 2004.

[3] N. Bonod, S. Enoch, L. F. Li, E. Popov, and M. Neviere, "Resonant optical transmission through thin metallic films with and without holes," Optics Express, vol. 11, pp. 482-490, 2003.

[4] B. F. Bai, L. F. Li, and L. J. Zeng, "Experimental verification of enhanced transmission through two-dimensionally corrugated metallic films without holes," Optics Letters, vol. 30, pp. 2360-2362, 2005.

[5] T. W. Ebbesen, H. J. Lezec, H. F. Ghaemi, T. Thio, and P. A. Wolff, "Extraordinary optical transmission through sub-wavelength hole arrays," Nature, vol. 391, pp. 667-669, 1998.

[6] H. J. Lezec, A. Degiron, E. Devaux, R. A. Linke, and e. al, "Beaming light from a subwavelength aperture," Science, vol. 297, pp. 820-822, 2002 .

[7] I. Avrutsky, Y. Zhao, and V. Kochergin, "Surface-plasmon-assisted resonant tunneling of light through a periodically corrugated thin metal film," Optics Letters, vol. 25, pp. 595-597, 2000.

[8] J. A. Porto, F. J. Garcia-Vidal, and J. B. Pendry, "Transmission resonances on metallic gratings with very narrow slits," Physical Review Letters, vol. 83, pp. 2845-2848, 1999

[9] E. Popov, M. Neviere, S. Enoch, and R. Reinisch, "Theory of light transmission through subwavelength periodic hole arrays," Physical Review B, vol. 62, pp. 16100-16108, 2000.

[10] L. Martin-Moreno, F. J. Garcia-Vidal, H. J. Lezec, K. M. Pellerin, T. Thio, J. B. Pendry, and T. W. Ebbesen, "Theory of extraordinary optical transmission through subwavelength hole arrays," Physical Review Letters, vol. 86, pp. 1114-1117, 2001.

[11] M. G. Moharam, E. B. Grann, D. A. Pommet, and T. K. Gaylord, "Formulation for Stable and Efficient Implementation of the Rigorous Coupled-Wave Analysis of Binary Gratings," Journal of the Optical Society of America a-Optics Image Science and Vision, vol. 12, pp. 1068-1076, 1995.

[12] http://www.gsolver.com.

[13] P. B. Johnson and R. W. Christy, "Optical Constants of the Noble Metals," Physical Review B, vol. 6, pp. 4370-4379, 1972.

[14] H. Raether, Surface plasmons on smooth and rough surfaces and on gratings: Berlin ; New York : Springer-Verlag, 1988.

[15] J. M. Steele, C. E. Moran, A. Lee, C. M. Aguirre, and N. J. Halas, "Metallodielectric gratings with subwavelength slots: optical properties," Physical Review B, vol. 68, pp. art. no.-205103, 2003. 